\title{
Bio-inspired hydrogel-based bandage with robust adhesive and antibacterial abilities for skin closure
}

\author{
Penghui Wang ${ }^{1 \dagger}$, Yajie $\mathrm{Pu}^{1 \dagger}$, Yanhan Ren ${ }^{4}$, Shuai Liu ${ }^{3}$, Rong Yang ${ }^{1}$, Xiaoyan Tan ${ }^{1,2}$, Wenjie Zhang ${ }^{1}$, \\ Tianqi $\mathrm{Shi}^{1}$, Shuang $\mathrm{Li}^{1}$ and $\mathrm{Bo} \mathrm{Chi}^{1,2^{*}}$
}

\begin{abstract}
Although conventional suturing techniques are commonly used in assisting wound closure, they do pose limited conduciveness and may lead to secondary injury to wound tissues. Inspired by marine organism mussels, we designed and manufactured a bio-inspired hydrogel-based bandage with tough wet tissue adhesion to substitute traditional surgical suture, accelerate wound healing and avoid infection. Poly $(\gamma$-glutamic acid) was modified with 3,4-dihydroxyphenylalanine and glycidyl methacylate, then introduced into the acrylic acid-co-acrylamide hydrogel matrix with robust mechanical properties. The hydrogel bandage showed strong chemical linkage adhesion $(70 \pm 2.1 \mathrm{kPa})$, which is 2.8 times that of commercial tissue adhesive fibrin glue $(25 \pm 2.2 \mathrm{kPa})$. The hydrogel bandage can not only maintain the self-stability, but is also capable of self-tuning adhesive strength in the range of $14-70 \mathrm{kPa}$ to achieve different adhesion effects by tuning constituent ratio. The bandage has desirable compression properties $(0.7 \pm 0.11 \mathrm{MPa})$ and tensile elongation (about 25 times), which ensures its resistance to damages, especially in joint spaces. Secondly, the bandage was endowed with antioxidant and endogenous broad-spectrum antibacterial properties with its catechol structure. Results also demonstrated excellent cell compatibility and blood compatibility, certifying its eligible biological safety profile. In a rat full-thickness cutaneous deficiency model, we can clearly observe that the bandage possesses the ability to promote wound healing (only need 6 days). Above all, this research provides a new strategy for the emergency treatment of liver hemostasis and myocardial repair during disaster rescue.
\end{abstract}

Keywords: hydrogel-based bandage, adhesion, antibacterial, antioxidant, skin closure

\section{INTRODUCTION}

Skin is the very first barrier of the human body; once damaged, skin and its underlying soft tissue will initiate various types of inflammatory responses to ward off bacterial infection [1], and induce granulation tissue formation [2]. However, granulation tissue has low tensile strength, which can lead to wound tear under stress [1]. At present, the gold standard for wound closure is achieved by primary intention such as suturing and stapling $[1,3]$. Although the above wound closure strategy can effectively achieve skin wound closure and skin incision sealing, there are many problems with the traditional surgical suture strategy, including the need for anesthesia [3], secondary wound injury, wound infection [4], longer healing time [5], and significant scar formation [2], which is not conducive during disaster scenarios, emergency rescue, and post-traumatic recoveries [1,6,7]. There is an urgent need to develop a new type of tissue adhesive for first aid, with the goals of reducing bacterial infection, free radical production and wound healing time [8].

With the recent breakthrough in the development of biomedical materials, a number of tissue adhesives have been used in auxiliary wound closure therapy, replacing the need for surgical sutures [1]. The latest clinical biological adhesives mainly include fibrin glue [9], cyanoacrylate glue [10,11], and serum albumin/glutaraldehyde [12]. Although they all can assist in tissue closure, their adhesion abilities are limited due to failure to maintain dry wound surfaces. Research has also shown that these adhesives often lead to longer wound healing span and higher infection rates due to poor mechanical properties, significantly limiting their clinical application $[13,14]$. In response to the above problems, a variety of hydrogel tissue adhesives (polyethylene glycol [15], alginate [16], acrylic acid (Aa) and other composite hydrogel tissue adhesives) have been researched and developed [17-20], which effectively enhanced the adhesion of the tissue interface and reduced the phase transformation time of the hydrogel. However, due to the complex preparation system and mechanical deformation caused by limb movement, it is difficult to ensure a stable connection between the adhesives and the skin interface $[5,9]$. Therefore, tissue adhesives with superior performance are needed to effectively achieve tissue adhesions and avoid interference of mechanical activities.

In nature, marine organisms such as mussels can firmly adhere to the surface of various organic and inorganic substances through the secretion of byssus in the body even in humid, dynamic, and saline-alkaline marine environments [21-23]. Studies have shown that the excellent adhesion properties of mussels come from the mussel adhesive protein of foot silk [21]. At present, six kinds of adhesion proteins have been identified from mussel foot silk, and each of them contains dopa

\footnotetext{
${ }^{1}$ State Key Laboratory of Materials-Oriented Chemical Engineering, Nanjing Tech University, Nanjing 211816, China

2 Jiangsu National Synergetic Innovation Center for Advanced Materials, Nanjing Tech University, Nanjing 211816, China

${ }^{3}$ School of Chemical Engineering, Nanjing University of Science and Technology, Nanjing 210094, China

${ }^{4}$ Chicago Medical School, Rosalind Franklin University of Medicine and Science, North Chicago, Illinois, 60064, USA

$\dagger$ These authors contributed equally to this work.

* Corresponding author (email: chibo@njtech.edu.cn)
} 
amino acids [24-26]. As their functional group, catechol can interact with various surfaces through multiple molecular interactions [27], including non-covalent interaction (hydrogen bond, $\pi$-hydrogen bond stacking, quinone hydrogen charge transfer complexation, etc.) and covalent anchoring interaction (Michael addition, aromatic copolymerization, etc.) to achieve broad-spectrum and high-strength interface bonding $[22,23,28,29]$, which provides a new idea for designing tissueintegrated hydrogels with excellent performance. However, the problem of microbial invasion caused by the weak integration between the hydrogel and wound, and the wound infection caused by the bacterial incubated in moist wound environment urgently need to be addressed [13,22,30]. In recent years, most solutions are based on hydrogel as the carrier to deliver antibacterial or silver nanoparticles [31-33]. However, inadequate stewardship of antibiotics can lead to antibiotics resistance, and the safety of silver ions is not clear [4,34]. Therefore, there is an urgent need to develop a hydrogel with high efficiency and endogenous antibacterial properties, good biocompatibility, tissue integration and mechanical properties.

Herein, based on the bionic strategy of mussels, poly $(\gamma$-glutamic acid) ( $\gamma$-PGA) was functionally modified with 3,4-dihydroxyphenylalanine (DA) and glycidyl methacrylate (GMA) to prepare and develop poly(amino acid) hydrogel-based bandage with tough mechanical properties and eligible biocompatibility. It has the capacities to assist in tissue integration while reducing infection and oxidative damages. It can also be used as a skin regeneration scaffold to recruit autologous cells (especially stem cells) and achieve rapid skin healing [4]. $\gamma$-PGA is a type of natural polymer material with commendable biocompatibility $[35,36]$. In previous work, we have proved that $\gamma$-PGA has the potential to promote wound healing, cellular adhesion, migration and differentiation. Optimization to wet tissue adhesion of hydrogel-based bandage is the main goal of this study, since the adhesion of wet tissue is a hot spot but difficult task of current research. In this study, GMA was introduced to improve the cohesion of the hydrogel-based bandage, and balance its adhesion strength and structural stability on the micro scale. We also explored the biological properties of liquid bandage by evaluating its biological safety, antibacterial properties, antioxidant properties and skin healing properties. Our findings provide a new solution and strategy for stat control of liver hemostasis and myocardial repair in emergent scenarios.

\section{RESULTS AND DISCUSSION}

\section{Preparation and characterization of $\gamma$-PGA-DA-GMA}

Inspired by the mussel, various functionalized hydrogel adhesives based on the catechol group have been developed to cope with the combination of tissue interfaces under wet conditions. In view of this, we designed and prepared a broad-spectrum antimicrobial hydrogel-based bandage for wet tissue adhesion. For the sake of the integration of the interface, we introduced DA into the $\gamma$-PGA chains with creditable biocompatibility in the presence of 1-ethyl-3-(3-dimethylaminopropyl-carbodiimide) hydrochloride (EDC)/ $N$-hydroxysuccinimide (NHS), to provide a stable and tough wet tissue adhesion for the hydrogel. The synthesis route is shown in Fig. S1. The ${ }^{1} \mathrm{H}$ nuclear magnetic resonance (NMR) spectrum (Fig. 1c) shows the catechol absorption peak of $\gamma$-PGA-DA at 6.5-7.2 ppm, which indicates the binding of DA to the main chain of $\gamma$-PGA. Compared with unmodified $\gamma$-PGA, both $\gamma$-PGA-DA and DA have obvious absorption peaks at $280 \mathrm{~nm}$ in the ultraviolet-visible (UV-Vis) wavelength scanning (Fig. 1d), which further confirms the successful modification with DA $[22,23,37]$. At the same time, we continued to introduce double-bond groups into the polymer chain, to enhance the cohesive force of the network and reinforce the adhesive strength and stability of the liquid bandage through the covalent bonding with GMA. The ${ }^{1} \mathrm{H}$ NMR spectrum of $\gamma$-PGA-DA-GMA shows the characteristic absorption peak of the double bond at 5.5-6.2 ppm, which verifies the successful modification with GMA $[29,38]$.

\section{Preparation and characterization of hydrogels}

Different concentrations of $\gamma$-PGA-DA-GMA were copolymerized with $\mathrm{Aa}$ and acrylamide (Am) monomers to construct hydrogels $(\gamma$-PDM). Schematic diagrams of hydrogel formation are shown in Fig. 1a, e. In order to achieve the intelligent response of hydrogel-based bandages, photoinitiators were used to realize the conversion of solution to gel of the polymer under UV irradiation (Fig. 1b). Gelation time is the linchpin to the production process and clinical application, which should be short enough to ensure the convenience [18,39-42]. The vial inversion method was adopted to evaluate the effect of Am and $\gamma$-PGA biopolymer with a series of contents on the gelation time (Fig. 2a). The results showed that all samples could be crosslinked under UV irradiation of $365 \mathrm{~nm}(2-30 \mathrm{~min})$, and the gelation time could be shortened from $15 \pm 0.60$ to $2 \pm 0.19 \mathrm{~min}$ $(\mathrm{Am}=10 \%)$ with the increasing concentration of Am. However, with the addition of $\gamma$-PGA-DA, the gelation time was extended to $30 \pm 1.3 \mathrm{~min}$. This is mainly because the catechol group has certain antioxidant capacity. Therefore, with the introduction of DA, the double-bond free radicals produced by UV light will be quenched partly. On the other hand, because $\gamma$-PGA is a flexible chain, it will hinder the polymerization of small molecules of Aa and Am, thus prolonging the gelation time of hydrogels. In order to reduce this adverse effect, $\gamma$-PGA-DA-GMA was prepared. With the increase of $\gamma$-PGA concentration, the gelation time was shortened to $2( \pm 0.66)-5.6( \pm 0.67) \mathrm{min}$, which preferably meets the goal. The differences were possibly attributed to the introduction of double bonds which could build a "bridge" for the polymerization of small molecules and accelerate the gelation process, weakening the free radical quenching caused by the existence of $\gamma$-PGA-DA, which manifests its potential as a candidate for tissue adhesive.

\section{Evaluation of the mechanical properties of hydrogels}

Hydrogel-based bandages are usually exposed to the risk of being damaged. In this study, the mechanical properties of hydrogels were evaluated by testing their compression and tensile strengths. The experimental results demonstrated that the increase of the Am concentration increased the compression modulus (Fig. 2e and Fig. S2a) to $1.66 \pm 0.15 \mathrm{MPa}$, but the addition of $\gamma$-PGA-DA decreased the compression modulus to $0.2 \pm 0.14 \mathrm{MPa}$ (Fig. 2f). The possible reason for this behavior is as follows: with the introduction of the $\gamma$-PGA-DA polymer, the double-bond free radicals generated by UV irradiation were consumed, and the polymerization of the small molecules of $\mathrm{Aa}$ and Am was blocked, thus reducing the modulus of compression, which is consistent with the prolongation of the gelation time. Similarly, double bonds were chosen to solve this problem. The results showed that this modification could effectively avert 


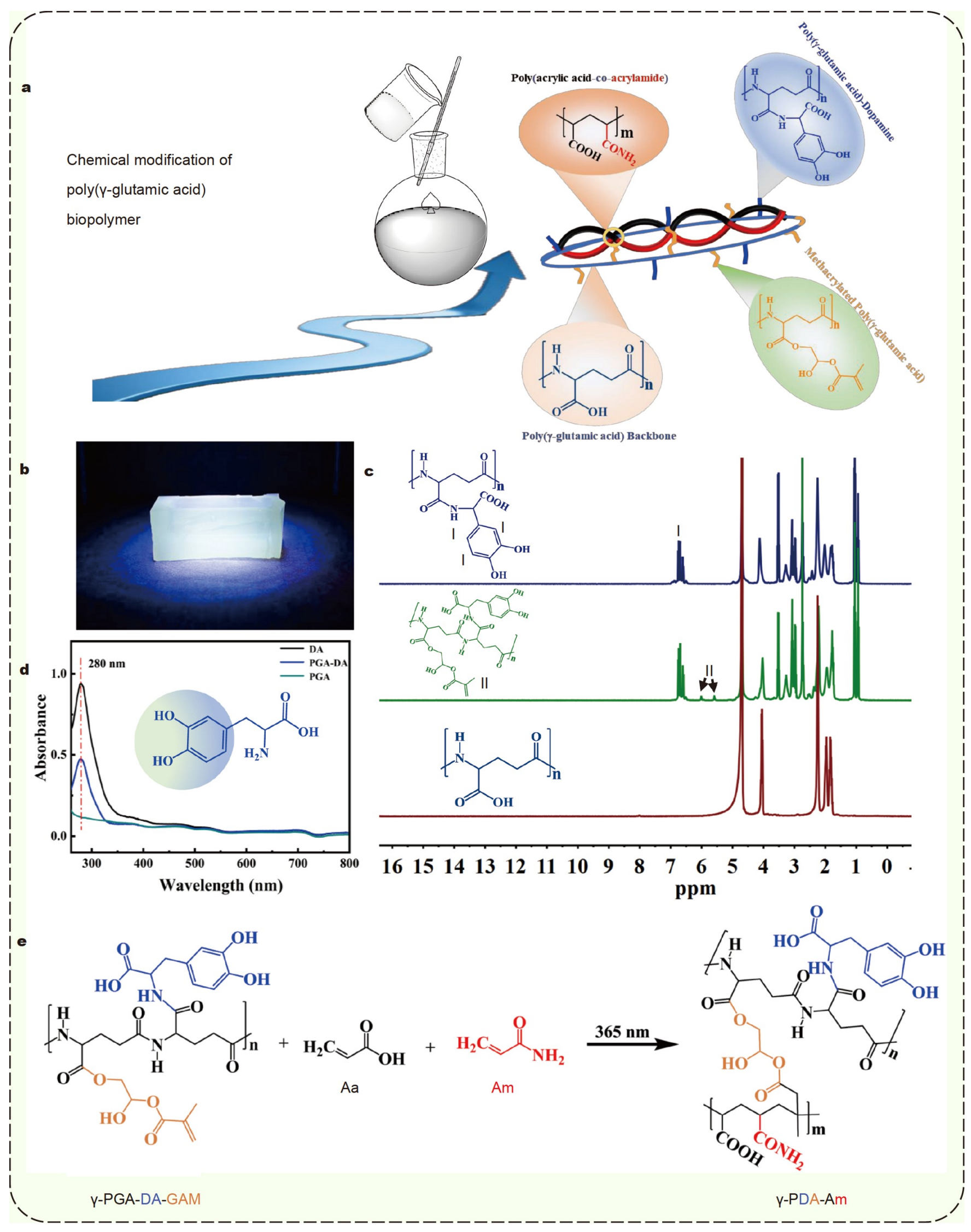

Figure 1 Design, preparation, and characterization of the adhesive hydrogels. (a) Schematic diagram of the chemical modification of $\gamma$-PGA. (b) Schematic diagram of the UV curing of adhesive hydrogels. (c) ${ }^{1} \mathrm{H}$ NMR and (d) UV-Vis of the as-synthesized polymers. (e) Schematic diagram of the UV curing principle of the adhesive hydrogels. 


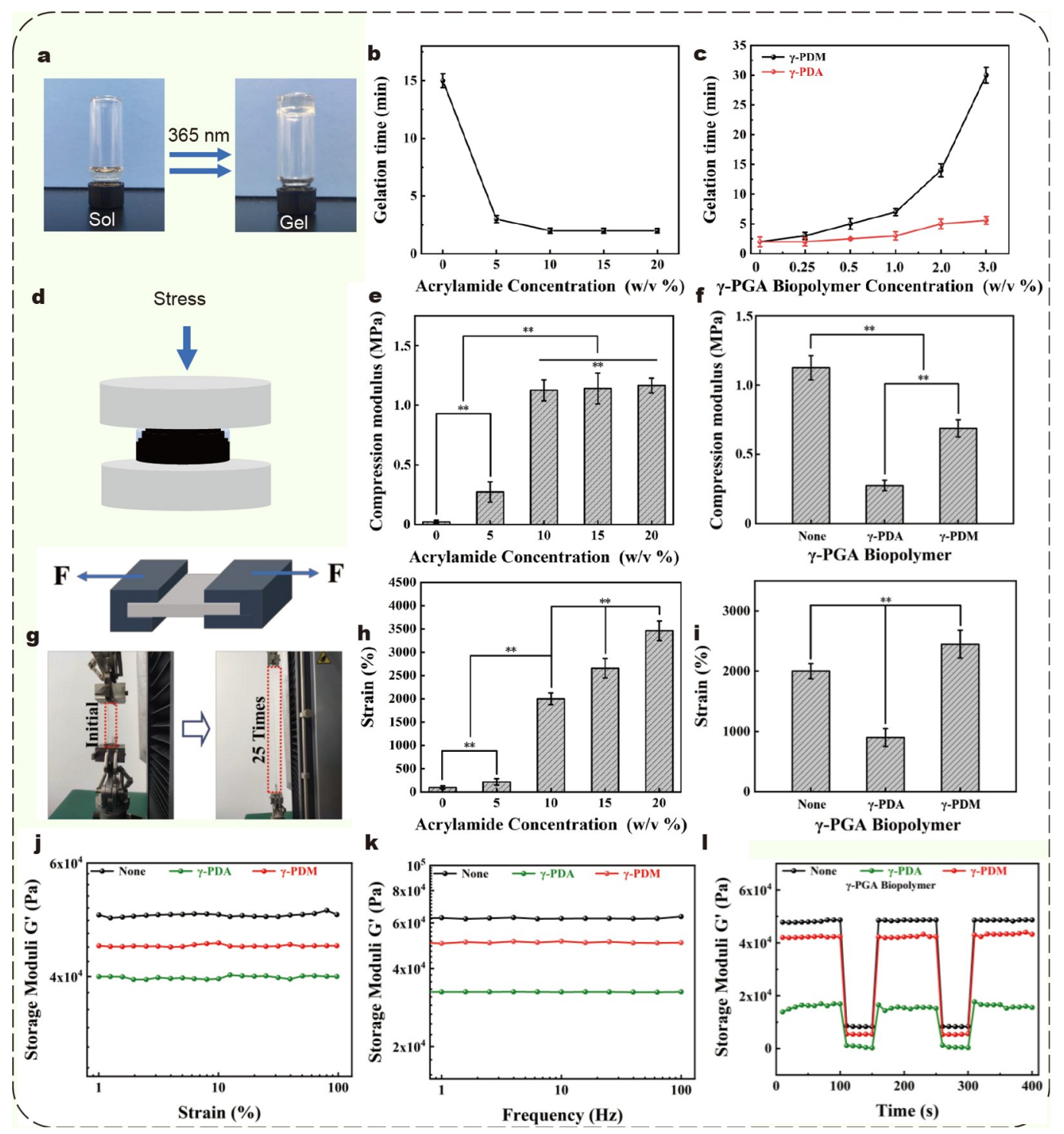

Figure 2 Characterization of the mechanical properties of the $\gamma$-PGA hydrogels. (a) Transition from the sol state of $\gamma$-PGA hydrogels to the gel state. UV curing times of (b) simple acrylic ester hydrogels and (c) $\gamma$-PGA hydrogels. (d) Schematic diagram of the compression experiment and compressive modulus of (e) simple acrylic ester hydrogels and (f) the $\gamma$-PGA hydrogels. (g) Schematic diagram of the tensile experiment and tensile properties of (h) simple acrylic ester hydrogels and (i) the $\gamma$-PGA hydrogels. Rheological properties of the $\gamma$-PGA hydrogels, (j) stress sweep, (k) frequency sweep, and (l) cyclic strain time sweep. $\gamma$-PDA hydrogels is the abbreviation of hydrogels prepared by combining $\gamma$-PGA-DA, Aa, and Am.

the drawback of low crosslinking density compared with the system without double bonds, and the compression modulus increased significantly to $0.7 \pm 0.11 \mathrm{MPa}$. Likewise, the decreasing trend of the compression modulus attributed to the restriction of polymer chains clearly slowed down. The tensile property test also presented the same tendency (Fig. $2 \mathrm{~g}-\mathrm{i}$ and Fig. S2b). The significant improvement in the mechanical properties is considered to be a result of the cohesive force enhancement of the hydrogel system. Besides, the catechol and carboxyl groups of the modified $\gamma$-PGA chain can also form intermolecular interactions, such as high-density intermolecular hydrogen bonds and $\pi-\pi$ stacking interaction, which improve the internal stability of the $\gamma$-PDM hydrogels $[16,28,40]$. Finally, to have some insight into the influence of intermolecular and intramolecular interactions on cohesion, rheological tests were further performed. As shown in Fig. 2j, k, the storage modulus of
$\gamma$-PDM hydrogels notably increased compared with that of $\gamma$ PDA hydrogels. The cyclic strain time sweep (Fig. 21 and Fig. S2c) reveals that all the samples exhibited a certain degree of recovery under the shear stress of $10 \%-500 \%$, which provided convenience for later practical applications $[5,28,43]$. Furthermore, the experiments have shown that the concentration of $\gamma$ PGA biopolymer can be regulated to respond to various stresses; moreover, it shows rapid and complete structural recovery when the stress is removed (Movie S1). This can be explained by the presence of $\mathrm{Aa}$ and Am copolymerization in acrylate hydrogel so that the system has strong compression and tensile properties. Even in the presence of the $\gamma$-PGA-DA polymer chain, the mechanical properties of the entire system are not impacted significantly when double bonds are introduced in the $\gamma$-PGADA polymer chain. The aim is to avoid the damage of the mechanical modulus of acrylate by the $\gamma$-PGA polymer chain 


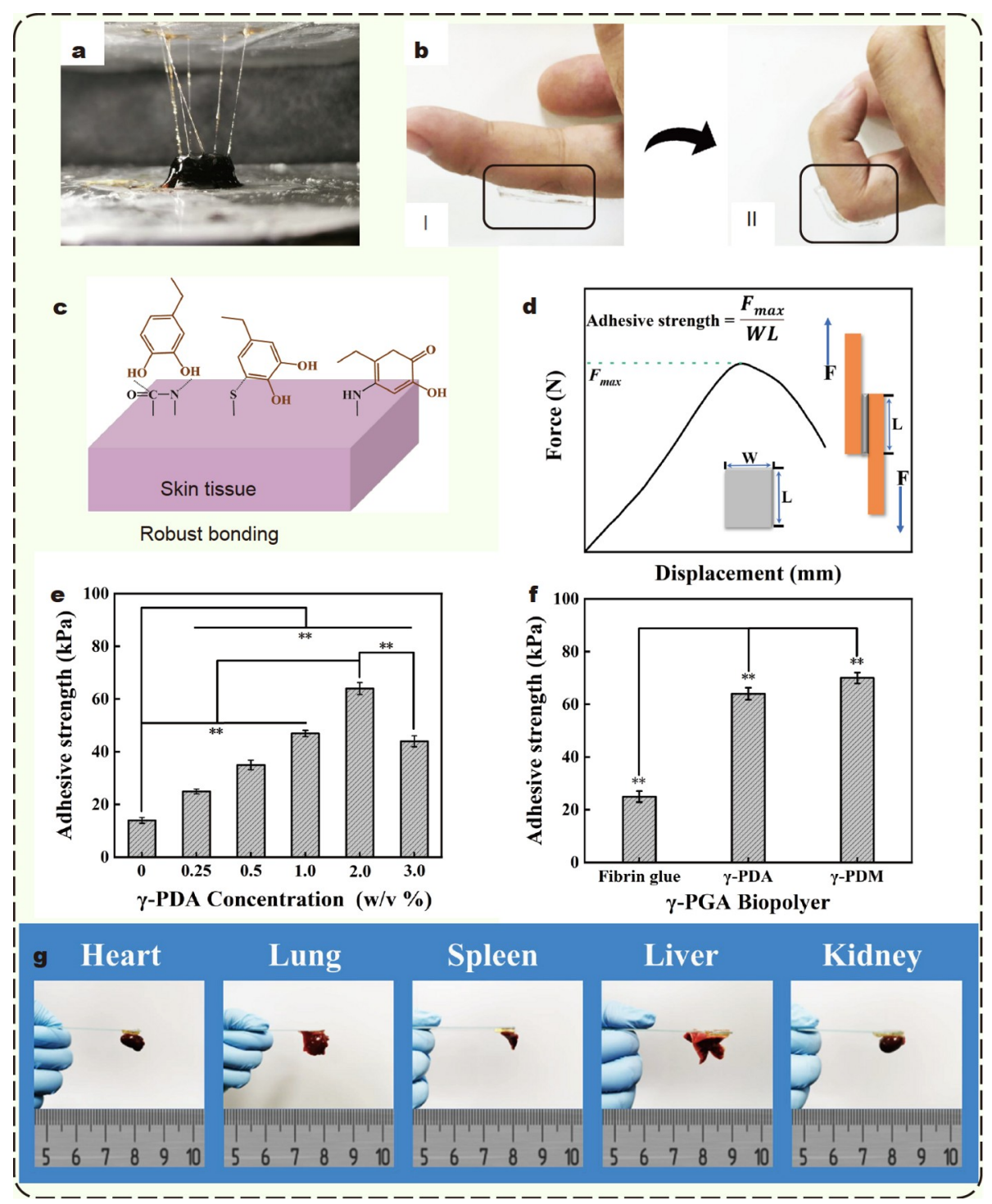

Figure 3 Evaluation of the in vitro adhesive performance of the $\gamma$-PGA hydrogels. (a) Adhesion behavior of the $\gamma$-PGA hydrogels. (b) Demonstration of the excellent tissue adhesion ability of the $\gamma$-PGA hydrogels. (c) Adhesion mechanism of the adhesive $\gamma$-PGA hydrogels. (d) Schematic diagram of the adhesion test of the $\gamma$-PGA hydrogels. Comparison of the adhesion strengths of (e) $\gamma$-PGA hydrogels with different $\gamma$-PDA concentrations and (f) adhesion strengths of different hydrogels. (g)Photographs of the adhesive hydrogels adhered to different tissues (e.g., heart, lung, spleen, liver, and kidney from rat).

through the improvement in the system cohesion. The results all confirmed that the $\gamma$-PDM hydrogel has the advantage of mechanical performance.

\section{Characterization of the adhesion ability of hydrogels}

The tissue adhesive properties of $\gamma$-PDM hydrogels were further investigated. First, it was confirmed that $\gamma$-PDM hydrogels have a similar adhesion pattern to mussel foot byssus (Fig. 3a), providing a certain adhesive strength for the integration of the wet tissue interface. In practical application, the joint is not easy to adhere to and easily falls off. Therefore, there is high demand for improving the wound bandage's adhesion ability. Thus, the liquid bandage was pasted on the joints of the fingers, adhering firmly to the skin even if the fingers repeatably bend and extend, which demonstrates its good adaptability and adhesion (Fig. 3b and Movie S2). The present study indicates that Aa can be bridged to the tissue interface through multiple hydrogen bonds. At the same time, catechol components can form stable chemical covalent bonds with various groups such as amino and thiol (Fig. 3c). Additionally, the benzene ring components can contribute to the tough adhesion through $\pi-\pi$ stacking and cation $-\pi$ physical interactions, eventually realizing the close combination of soft tissues $[16,18,19,44]$. Subsequently, the adhesion properties of porcine skin were evaluated. As can be seen from Fig. $3 \mathrm{~d}-\mathrm{f}$, with the increase of $\gamma$-PGA-DA content, the adhesion performance increased firstly but then dropped. The $2 \% \gamma$-PDA hydrogels showed the best adhesion strength, reaching $64 \pm 2.3 \mathrm{kPa}$. All groups showed an improved strength to the mere acrylic ester hydrogels $(14 \pm 1.1 \mathrm{kPa})$. Therefore, the relationship between adhesion strength and cohesion of hydrogels was investigated by introducing double bonds [28,36,45]. The results showed that $\gamma$-PDM hydrogels exhibited a higher adhesion than fibrin glue $(25 \pm 2.1 \mathrm{kPa}) \cdot \gamma$-PDA hydrogels also showed good adhesion but had no significant advantage compared with $\gamma$-PDM hydrogels $(70 \pm 2.1 \mathrm{kPa})$. The adhesion strength of $\gamma$-PDM hydrogels could also be approximately 
2.8 times that of commercial fibrin glue, confirming that the introduction of double bonds not only has no limit to the adhesion of $\gamma$-PDM hydrogels but also slightly improved it. This is the synergistic effect of cohesion and adhesion of the hydrogel system. Finally, the adhesion ability of various soft tissues was evaluated, revealing that $\gamma$-PDM hydrogels have good adhesion to the soft tissues of the heart, liver, spleen, lung, and kidney in mice (Fig. 3g). Simultaneously, a new type of tissue adhesive apparatus was prepared using a $\gamma$-PDM hydrogel-assisted chain apparatus (Fig. 5b and Movie S3) and demonstrated better adhesion behavior. In a word, the enhancement of the internal cohesion was observed; hydrogels not only improved adhesion but also improved the mechanical properties, allowing more suitable clinical applications.

\section{Characterization of the antioxidant ability of hydrogels}

In the period of wound healing, the production of a great number of free radicals seriously hinders the speed of wound healing. Therefore, the evaluation of the hydrogel dressing's ability to scavenge free radicals is crucial for reducing oxidative damages in the 3D microenvironment of skin tissue [24,46,47]. The antioxidant capacity of hydrogels was further evaluated by testing the 1,1-diphenyl-2-picrylhydrazyl radical 2,2-diphenyl-1(2,4,6-trinitrophenyl)hydrazyl (DPPH) scavenging capability. As showed in Fig. S3, by decreasing the $\gamma$-PGA-DA content, the scavenging rate of DPPH was up to $89.65 \% \pm 4.98 \%$. This is mainly because DA is a type of polyphenol compound; its phenolic hydroxyl can directly act on free radical-related enzymes to achieve the purpose of scavenging free radicals. Furthermore, through the synergistic effect with PGA, it can achieve a good antioxidant effect, suggesting that hydrogels can accelerate wound healing by inhibiting the production of free radicals in wound tissue, which indicates great potential in the application for skin dressing and other aspects.

\section{Assessment of the biological safety ability of hydrogels}

Excellent biocompatibility is also a prerequisite for the efficient use of tissue adhesives. Therefore, the 3-(4,5-dimethyl-2-thiazolyl)-2,5-diphenyl tetrazolium bromide (MTT) test (Fig. 4a) and live/dead stain evaluation (Fig. $4 \mathrm{~b}$ ) were used to evaluate the in vitro cytotoxicity of hydrogels. The cell viability of all groups was higher than $80 \%$ after incubation in $\gamma$-PDM hydrogelsconditioned medium for 1,2 , and 3 days, respectively. We speculate the decline in cell viability is due to the presence of Aa residues in the system on the one hand, and the presence of catechol groups on the cell membrane on the other hand, which leads to apoptosis of some cells. However, the system polyglutamic acid with excellent biocompatibility can improve the cell function and increase the cell viability, so the cell viability was maintained above $80 \%$. It can be seen from the staining

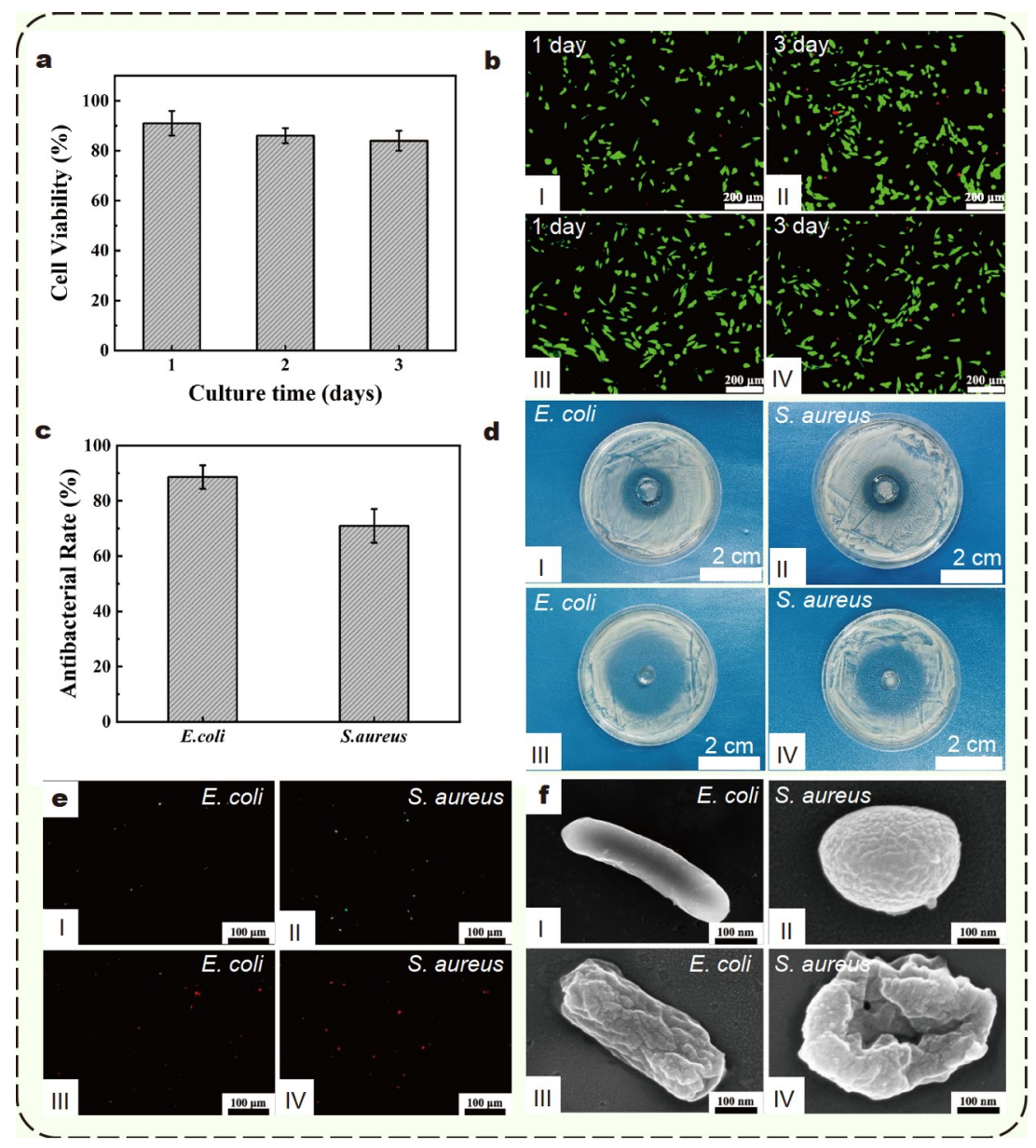

Figure 4 Evaluation of the in vitro biological performance of the adhesive $\gamma$-PGA hydrogels. (a) MTT test. (b) Live/dead stain evaluation of L929 cell. (I, II) DMEM; (III, IV) $\gamma$-PDM hydrogels. (c) Antibacterial rate for E. coli and S. aureus. (d) Inhibition zone method to evaluate the inhibition rate for E. coli and S. aureus. (I, II) Acrylic ester hydrogels; (III, IV) $\gamma$-PDM hydrogels. Live/dead stain evaluation of E. coli and S. aureus. (e) (I, II) Acrylic ester hydrogels; (III, IV) $\gamma$-PDM hydrogels. SEM evaluation of the micromorphology of E. coli and S. aureus. (f) (I, II) Acrylic ester hydrogels; (III, IV) $\gamma$-PDM hydrogels. 
pictures that the cells thrived and have the morphology of living cells (green). Meanwhile, compared with the culture medium of Dulbecco's modified eagle medium (DMEM), there was no significant difference in cell growth (Fig. 4b). This also indicated the superior cytocompatibility of $\gamma$-PDM hydrogels [48]. We continued to evaluate the blood compatibility of hydrogels. The results showed that (Fig. S4), compared with acrylic ester hydrogels, $\gamma$-PDA and $\gamma$-PDM showed better blood compatibility with hemolytic rates $<5 \%[1,17,49]$. All these results confirmed that $\gamma$-PDM hydrogels have higher biosecurity and clinical potentials for tissue repair and wide market prospects.

\section{Evaluation of the antibacterial properties of hydrogels}

The bacterial infection leads to the accumulation of exudate and the delay of the healing process. Desirable wound dressing should have excellent endogenous antibacterial activity, which can effectively avoid not only bacterial resistance but also antibiotics misuse. In this study, the antibacterial activity of hydrogels on E. coli and S. aureus was evaluated by the in vitro antibacterial activity test. The experimental results revealed that the $\gamma$-PDM hydrogels had commendable activity against $E$. coli $(88.6 \% \pm 4.25 \%)$ and $S$. aureus $(70.98 \% \pm 4.25 \%)$, exhibiting stronger bacteriostatic ability against E. coli (Fig. 4c). The experimental results of the inhibition zone (Fig. $4 \mathrm{~d}$ ) also confirmed that the bacteriostasis of $\gamma$-PDM hydrogels increased significantly compared with that of acrylic ester hydrogels. The inhibition radius of E. coli and S. aureus increased from $1.82 \pm$ 0.22 and $1.53 \pm 0.41 \mathrm{~cm}$ to $2.65 \pm 0.28$ and $2.11 \pm 0.62 \mathrm{~cm}$ (Table S1), respectively. The live/dead staining experiment of bacteria directly showed the effect of $\gamma$-PDM hydrogels on these two bacteria (Fig. 4e). For the scanning electron microscopy (SEM) image (Fig. 4f), it can be clearly seen that after applying
$\gamma$-PDM hydrogels, the bacterial surfaces changed from smooth and full to wrinkle or even dry to shrunken. The excellent antibacterial ability of the hydrogel is mainly due to the presence of the catechol functional group in the system, which can effectively act on the bacterial cell membrane, causing the bacterial cell membrane rupture and cytoplasm outflow, thereby achieving the sterilization effect. Moreover, the phenolic hydroxyl group of the catechol can also change the cell metabolism of microorganisms and easily bind to the active part of the enzyme, achieving the purpose of sterilization. Moreover, this endogenous antibacterial effect can ensure that there is no need to introduce other antibacterial reagents into the system to achieve antibacterial performance, which can effectively prevent the abuse of antibiotics and avoid the production of resistant bacteria $[50,51]$.

\section{Skin healing experiment in vivo}

To further evaluate the healing performance of the hydrogelbased bandage on skin incision (Fig. 5a), a minimally invasive medical device was prepared and used to evaluate the healing efficiency of the skin incision model (Fig. 5b). Compared with the control group (Fig. 5c), the wound healing rate of the skin incision in the non-contact auxiliary healing instrument was significantly faster (6 days). Histological examination (Fig. 5d) was used to evaluate the quality of the new tissue in the experimental group applied with $\gamma$-PDM hydrogels. The results of hematoxylin-eosin (H\&E) and Masson staining showed that the granulation and epithelial tissues were formed on the sixth day of the experiment group, and no obvious difference between the new skin tissue and the original skin tissue was observed. However, the control group clearly did not heal completely, exhibiting limited granulation and immature epithelial tissues.

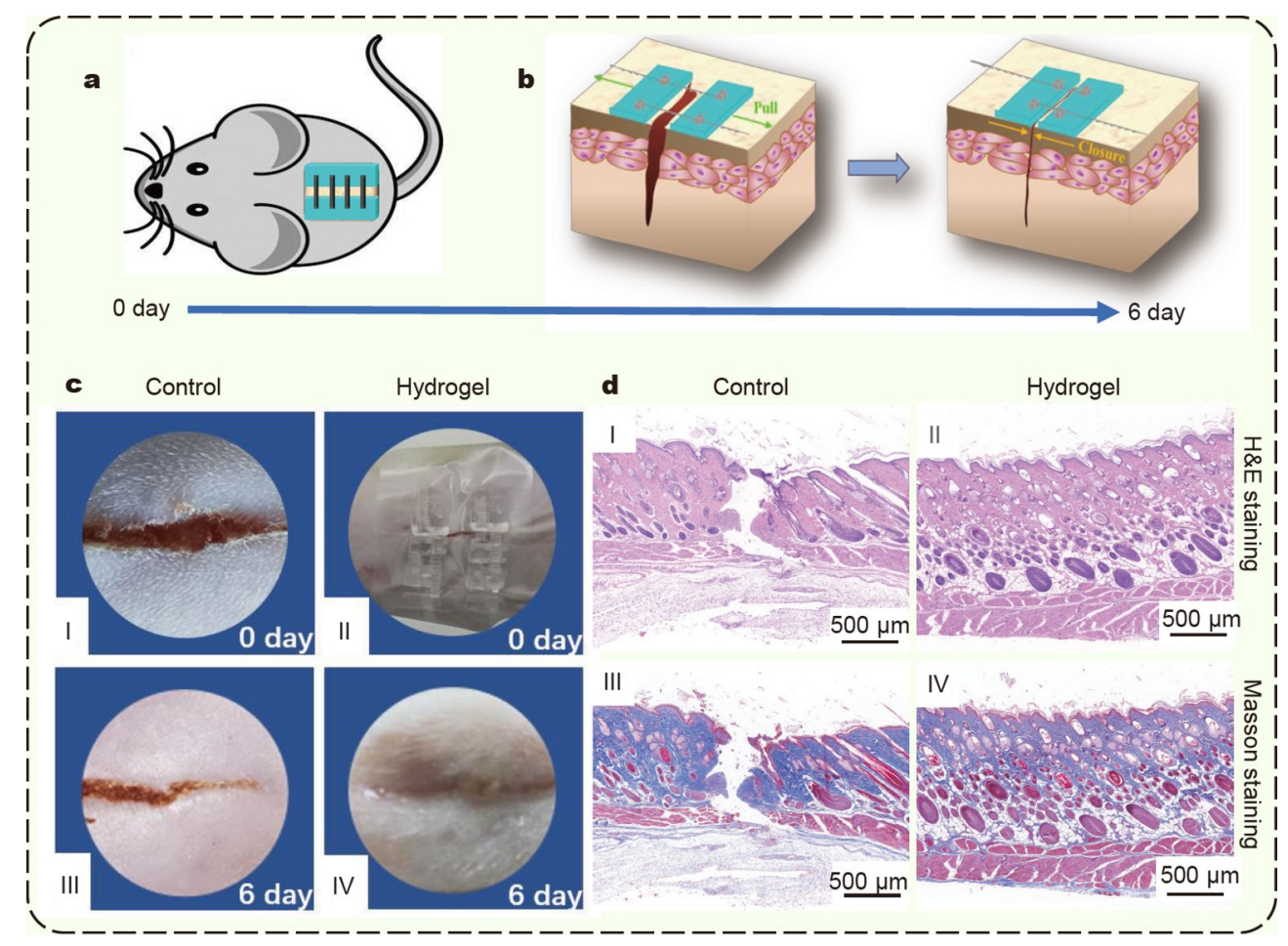

Figure 5 Evaluation of the in vivo adhesion performance of the adhesive $\gamma$-PGA hydrogels. (a) Schematic diagram of adhesion in vivo. (b) Schematic diagram of the adhesion behavior of the $\gamma$-PGA hydrogels. (c) Evaluation of the skin repair performance of the $\gamma$-PGA hydrogels at (I, II) 0 and (III, IV) 6 days. (d) H\&E and Masson staining images of the wound healing sites on the 6th day after the treatment. 
In short, $\gamma$-PDM hydrogels can effectively promote wound healing and induce the regeneration of tissue $[52,53]$.

\section{CONCLUSION}

In conclusion, a new type of skin non-invasion healing device based on $\gamma$-PGA was designed and prepared, which achieved robust adhesion properties and inherent antibacterial and antioxidant properties. The adhesive was also endowed with the function of effectively and rapidly promoting skin wound healing. First, $\gamma$-PGA-DA-GMA was incorporated into the acrylic Aa-co-Am hydrogel matrix to bridge the $\gamma$-PGA polymer chains with the monomers of Aa and Am through the bonding of double bonds. This process enhanced the cohesive force of $\gamma$ PDM hydrogels. These improvements proved to be effective in improving its adhesion on the skin surface and tissue interface. Additionally, $\gamma$-PDM hydrogels were used to repair skin incisions in vivo to replace conventional surgical sutures. The results proved that the $\gamma$-PDM hydrogels could firmly adhere to the skin tissue. Cooperating with the zipper system, it could achieve wound closure without trauma, accelerating the process of wound healing ( 6 days). Given this, we reckon that the hydrogel bandage adequately showed the broad spectrum of adhesion properties in the evaluation of wet soft tissue adhesion test and can be widely used in the fields of liver hemostasis, heart repair, plugging of blood vessels, organs, etc. It paves a new path and provides a new strategy for the current clinical operation problems, which has far-reaching significance during a surgical emergency.

Received 31 March 2021; accepted 31 May 2021; published online 12 August 2021

1 Ma Y, Yao J, Liu Q, et al. Liquid bandage harvests robust adhesive, hemostatic, and antibacterial performances as a first-aid tissue adhesive. Adv Funct Mater, 2020, 30: 2001820

2 Xu T, Yang R, Ma X, et al. Bionic poly $(\gamma$-glutamic acid) electrospun fibrous scaffolds for preventing hypertrophic scars. Adv Healthcare Mater, 2019, 8: 1900123

3 Ghobril C, Grinstaff MW. The chemistry and engineering of polymeric hydrogel adhesives for wound closure: A tutorial. Chem Soc Rev, 2015, 44: $1820-1835$

4 Qu J, Zhao X, Liang Y, et al. Antibacterial adhesive injectable hydrogels with rapid self-healing, extensibility and compressibility as wound dressing for joints skin wound healing. Biomaterials, 2018, 183: 185199

5 Zhao X, Liang Y, Huang Y, et al. Physical double-network hydrogel adhesives with rapid shape adaptability, fast self-healing, antioxidant and $\mathrm{NIR} / \mathrm{pH}$ stimulus-responsiveness for multidrug-resistant bacterial infection and removable wound dressing. Adv Funct Mater, 2020, 30: 1910748

6 Coventry CA, Vaska AI, Holland AJA, et al. Surgical procedures performed by emergency medical teams in sudden-onset disasters: A systematic review. World J Surg, 2019, 43: 1226-1231

7 Sever MS, Vanholder R, Lameire N. Management of crush-related injuries after disasters. N Engl J Med, 2006, 354: 1052-1063

8 Zhao H, Huang J, Li Y, et al. ROS-scavenging hydrogel to promote healing of bacteria infected diabetic wounds. Biomaterials, 2020, 258: 120286

9 Bouten PJM, Zonjee M, Bender J, et al. The chemistry of tissue adhesive materials. Prog Polym Sci, 2014, 39: 1375-1405

10 Barkan Y, Levinman M, Veprinsky-Zuzuliya I, et al. Comparative evaluation of polycyanoacrylates. Acta Biomater, 2017, 48: 390-400

11 Chang EI, Galvez MG, Glotzbach JP, et al. Vascular anastomosis using controlled phase transitions in poloxamer gels. Nat Med, 2011, 17:
$1147-1152$

12 Gaberel T, Borgey F, Thibon P, et al. Surgical site infection associated with the use of bovine serum albumine-glutaraldehyde surgical adhesive (BioGlue $\left.{ }^{\circledR}\right)$ in cranial surgery: A case-control study. Acta Neurochir, 2011, 153: 156-163

13 Cui C, Fan C, Wu Y, et al. Water-triggered hyperbranched polymer universal adhesives: From strong underwater adhesion to rapid sealing hemostasis. Adv Mater, 2019, 31: 1905761

14 Bai J, Wang R, Ju M, et al. Facile preparation and high performance of wearable strain sensors based on ionically cross-linked composite hydrogels. Sci China Mater, 2021, 64: 942-952

15 Bu Y, Zhang L, Sun G, et al. Tetra-PEG based hydrogel sealants for in vivo visceral hemostasis. Adv Mater, 2019, 31: 1901580

16 Li J, Celiz AD, Yang J, et al. Tough adhesives for diverse wet surfaces. Science, 2017, 357: 378-381

17 Long M, Zhang Y, Huang P, et al. Emerging nanoclay composite for effective hemostasis. Adv Funct Mater, 2018, 28: 1704452

18 Yuk H, Varela CE, Nabzdyk CS, et al. Dry double-sided tape for adhesion of wet tissues and devices. Nature, 2019, 575: 169-174

19 Wirthl D, Pichler R, Drack M, et al. Instant tough bonding of hydrogels for soft machines and electronics. Sci Adv, 2017, 3: e1700053

20 Park J, Chang C, Kwon D. Use of fibrin adhesive for preventing pharyngocutaneous fistula in total laryngectomy. Am J Otolaryngol, 2020, 41: 102674

21 Huang S, Liu Y, Zhao Y, et al. Flexible electronics: Stretchable electrodes and their future. Adv Funct Mater, 2019, 29: 1805924

22 Wang $\mathrm{R}, \mathrm{Li} \mathrm{J}$, Chen W, et al. A biomimetic mussel-inspired $\varepsilon$-poly- $l$ lysine hydrogel with robust tissue-anchor and anti-infection capacity. Adv Funct Mater, 2017, 27: 1604894

23 Liu S, Liu X, Ren Y, et al. Mussel-inspired dual-cross-linking hyaluronic acid/ $\varepsilon$-polylysine hydrogel with self-healing and antibacterial properties for wound healing. ACS Appl Mater Interfaces, 2020, 12: 27876-27888

24 Tang P, Han L, Li P, et al. Mussel-inspired electroactive and antioxidative scaffolds with incorporation of polydopamine-reduced graphene oxide for enhancing skin wound healing. ACS Appl Mater Interfaces, 2019, 11: 7703-7714

25 Han L, Lu X, Liu K, et al. Mussel-inspired adhesive and tough hydrogel based on nanoclay confined dopamine polymerization. ACS Nano, 2017, 11: 2561-2574

26 Han L, Liu K, Wang M, et al. Mussel-inspired adhesive and conductive hydrogel with long-lasting moisture and extreme temperature tolerance. Adv Funct Mater, 2018, 28: 1704195

27 Madhurakkat Perikamana SK, Lee J, Lee YB, et al. Materials from mussel-inspired chemistry for cell and tissue engineering applications. Biomacromolecules, 2015, 16: 2541-2555

28 Jung H, Kim MK, Lee JY, et al. Adhesive hydrogel patch with enhanced strength and adhesiveness to skin for transdermal drug delivery. Adv Funct Mater, 2020, 30: 2004407

29 Chen W, Wang R, Xu T, et al. A mussel-inspired poly( $\gamma$-glutamic acid) tissue adhesive with high wet strength for wound closure. J Mater Chem B, 2017, 5: 5668-5678

30 Cui $\mathrm{C}, \mathrm{Wu} \mathrm{T}$, Chen $\mathrm{X}$, et al. A Janus hydrogel wet adhesive for internal tissue repair and anti-postoperative adhesion. Adv Funct Mater, 2020, 30: 2005689

31 Zhao Y, Li Z, Song S, et al. Skin-inspired antibacterial conductive hydrogels for epidermal sensors and diabetic foot wound dressings. Adv Funct Mater, 2019, 29: 1901474

32 Zheng K, Balasubramanian P, Paterson TE, et al. Ag modified mesoporous bioactive glass nanoparticles for enhanced antibacterial activity in 3D infected skin model. Mater Sci Eng-C, 2019, 103: 109764

33 Guo F, Liu C, Han R, et al. Bio-inspired anisotropic polymeric heart valves exhibiting valve-like mechanical and hemodynamic behavior. Sci China Mater, 2020, 63: 629-643

34 Chen H, Cheng J, Ran L, et al. An injectable self-healing hydrogel with adhesive and antibacterial properties effectively promotes wound healing. Carbohyd Polym, 2018, 201: 522-531

35 Wang $\mathrm{X}$, Shi $\mathrm{H}$, Tang $\mathrm{H}$, et al. Electrostatic assembly functionalization of poly ( $\gamma$-glutamic acid) for biomedical antibacterial applications. J 
Mater Sci Tech, 2020, 59: 14-25

36 Wang P, Zhang W, Yang R, et al. Biomimetic poly $(\gamma$-glutamic acid) hydrogels based on iron (III) ligand coordination for cartilage tissue engineering. Int J Biol Macromolecules, 2020, 167: 1508-1516

37 Hasani-Sadrabadi MM, Sarrion P, Pouraghaei S, et al. An engineered cell-laden adhesive hydrogel promotes craniofacial bone tissue regeneration in rats. Sci Transl Med, 2020, 12: eaay6853

38 Zeng W, Hu W, Li H, et al. Preparation and characterization of poly $(\gamma-$ glutamic acid) hydrogels as potential tissue engineering scaffolds. Chin J Polym Sci, 2014, 32: 1507-1514

39 Huang J, Liu Y, Chi X, et al. Programming electronic skin with diverse skin-like properties. J Mater Chem A, 2021, 9: 963-973

40 Li J, Illeperuma WRK, Suo Z, et al. Hybrid hydrogels with extremely high stiffness and toughness. ACS Macro Lett, 2014, 3: 520-523

41 Hong Y, Zhou F, Hua Y, et al. A strongly adhesive hemostatic hydrogel for the repair of arterial and heart bleeds. Nat Commun, 2019, 10: 2060

42 Tang P, Yan H, Chen L, et al. Anisotropic nanocomposite hydrogels with enhanced actuating performance through aligned polymer networks. Sci China Mater, 2020, 63: 832-841

43 Chaudhuri O, Cooper-White J, Janmey PA, et al. Effects of extracellular matrix viscoelasticity on cellular behaviour. Nature, 2020, 584: 535-546

44 Deng X, Huang B, Wang Q, et al. A mussel-inspired antibacterial hydrogel with high cell affinity, toughness, self-healing, and recycling properties for wound healing. ACS Sustain Chem Eng, 2021, 9: 30703082

45 Yang $\mathrm{Y}, \mathrm{Xu} \mathrm{T}$, Zhang Q, et al. Biomimetic, stiff, and adhesive periosteum with osteogenic-angiogenic coupling effect for bone regeneration. Small, 2021, 17: 2006598

46 Ge W, Cao S, Shen F, et al. Rapid self-healing, stretchable, moldable, antioxidant and antibacterial tannic acid-cellulose nanofibril composite hydrogels. Carbohydrate Polyms, 2019, 224: 115147

47 Turabee MH, Thambi T, Lee DS. Development of an injectable tissue adhesive hybrid hydrogel for growth factor-free tissue integration in advanced wound regeneration. ACS Appl Bio Mater, 2019, 2: 25002510

48 Sun X, Lang Q, Zhang H, et al. Electrospun photocrosslinkable hydrogel fibrous scaffolds for rapid in vivo vascularized skin flap regeneration. Adv Funct Mater, 2017, 27: 1604617

49 Wang L, Zhang X, Yang K, et al. A novel double-crosslinking-doublenetwork design for injectable hydrogels with enhanced tissue adhesion and antibacterial capability for wound treatment. Adv Funct Mater, 2019, 30: 1904156

50 He J, Shi M, Liang Y, et al. Conductive adhesive self-healing nanocomposite hydrogel wound dressing for photothermal therapy of infected full-thickness skin wounds. Chem Eng J, 2020, 394: 124888

51 Zhao X, Wu H, Guo B, et al. Antibacterial anti-oxidant electroactive injectable hydrogel as self-healing wound dressing with hemostasis and adhesiveness for cutaneous wound healing. Biomaterials, 2017, 122: 3447

52 Zhao X, Sun X, Yildirimer L, et al. Cell infiltrative hydrogel fibrous scaffolds for accelerated wound healing. Acta Biomater, 2017, 49: 66-77

53 Zhao X, Lang Q, Yildirimer L, et al. Photocrosslinkable gelatin hydrogel for epidermal tissue engineering. Adv Healthcare Mater, 2016, 5: 108118

Acknowledgements This work was supported by the National Natural Science Foundation of China (31771049), the Foundation of Key R\&D Project of Jiangsu Province (BE2018731), the Research Foundation of State Key Laboratory of Materials-Oriented Chemical Engineering (ZK201806, KL18-06 and ZK201606), the Six Talent Peaks Project of Jiangsu Province (SWYY-046), the Natural Science Foundation of Jiangsu Province (BK20200682) and the Postgraduate Research \& Practice Innovation Program of Jiangsu Province (SJCX20_0408).

Author contributions Wang $\mathrm{P}$ and $\mathrm{Pu} \mathrm{Y}$ designed and performed the experiments, and wrote the paper; Yang $\mathrm{R}$ and Shi $\mathrm{T}$ completed the data curation; Zhang W and Liu S revised and edited the paper; Ren Y and Li S validated the results; Tan $\mathrm{X}$ provided the experimental resources; Chi B proposed the concept and supervised this study. All authors contributed to the general discussion and revision of the manuscript.

Conflict of interest The authors declare that they have no conflict of interest.

Supplementary information Experimental details and supporting data are available in the online version of the paper.

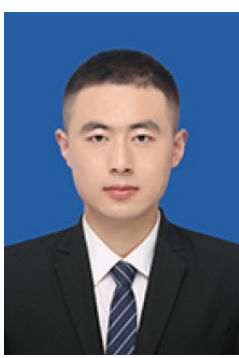

Penghui Wang is studying for his Master's degree at the State Key Laboratory of Materials-Oriented Chemical Engineering, College of Food Science and Light Industry, Nanjing Tech University. His research focuses on the construction of biodegradable biomedical polymers based on biomimetic strategies.

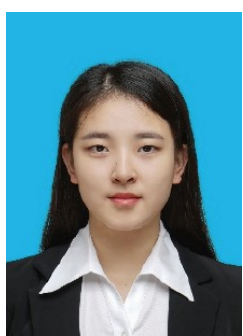

Yajie Pu is studying for her Master's degree at the State Key Laboratory of Materials-Oriented Chemical Engineering, College of Food Science and Light Industry, Nanjing Tech University. Her research focuses on the repair and treatment of difficult-to-heal wounds.

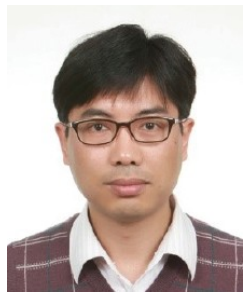

Bo Chi is a research fellow at the State Key Laboratory of Materials-Oriented Chemical Engineering, College of Food Science and Light Industry, Nanjing Tech University. He is currently engaged in basic and applied research in the field of biomaterials-medicalindustrial transformation, including biomedical materials, advanced functional soft materials; regulation of stem cell differentiation on biomaterial interfaces, and the application of biomedical materials in regenerative medicine.

\section{基于生物仿生策略的高性能皮肤拉链的制备及其抗 菌性能研究}

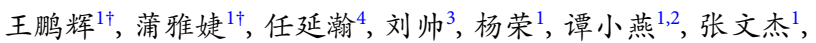
史天琪 ${ }^{1}$, 李霜 ${ }^{1}$, 迟波 ${ }^{1,2 *}$

摘要 传统的缝合技术易导致伤口组织继发性损伤，不利于皮肤伤口 愈合. 受海洋生物贻贝的启发, 我们设计并构筑了一种具有湿组织黏附 性能的仿生水凝胶朋带, 以替代传统的外科缝线, 加速伤口愈合, 防止 感染. 首先, 将 3,4 -二羟基苯丙氨酸和甲基丙烯酸缩水甘油酯改性的 $\gamma$ 聚谷氨酸引入到力学性能良好的丙烯酸酯水凝胶基质中, 构建具有优 异压缩性能 $(0.7 \pm 0.11 \mathrm{MPa}$ ) 和拉伸强度(约 25 倍)的水凝胶朋带, 以规 避其在肢体运动过程中 (尤其是关节部位) 受到损坏. 此外, 水凝胶朋带 显示出良好的组织黏合性能 $(70 \pm 2.1 \mathrm{kPa})$, 是市售组织黏合剂纤维蛋白 胶的 2.8 倍 $(25 \pm 2.2 \mathrm{kPa})$, 而且还可以通过调整水凝胶成分的组成, 使其 黏附性能在14-70 kPa的范围内可调. 其次, 由于体系中邻苯二酚基团的 存在, 使水凝胶绷带兼具优异的抗氧化和内源广谱抗菌性能. 结果还显 示水凝胶具有良好的生物安全性, 以及优异的促伤口愈合能力 (仅6天), 有望为灾后救援工作中的创伤紧急止血提供新的治疗策略. 